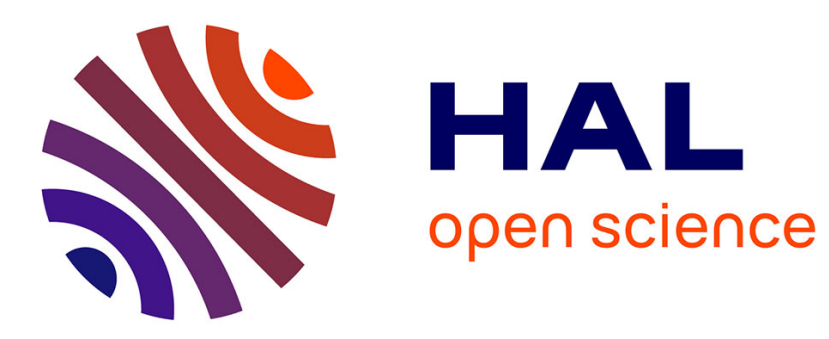

\title{
Leptons in near earth orbit
}

J. Alcaraz, B. Alpat, G. Ambrosi, H. Anderhub, L. Ao, A. Arefiev, P.

Azzarello, E. Babucci, Luca Baldini, M. Basile, et al.

\section{To cite this version:}

J. Alcaraz, B. Alpat, G. Ambrosi, H. Anderhub, L. Ao, et al.. Leptons in near earth orbit. Physics Letters B, 2000, 484, pp.10-22. 10.1016/S0370-2693(00)00588-8 . in2p3-00005529

\section{HAL Id: in2p3-00005529 https://hal.in2p3.fr/in2p3-00005529}

Submitted on 6 Jul 2000

HAL is a multi-disciplinary open access archive for the deposit and dissemination of scientific research documents, whether they are published or not. The documents may come from teaching and research institutions in France or abroad, or from public or private research centers.
L'archive ouverte pluridisciplinaire HAL, est destinée au dépôt et à la diffusion de documents scientifiques de niveau recherche, publiés ou non, émanant des établissements d'enseignement et de recherche français ou étrangers, des laboratoires publics ou privés. 


\title{
Leptons in Near Earth Orbit
}

\author{
The AMS Collaboration
}

\begin{abstract}
The lepton spectra in the kinetic energy ranges 0.2 to $40 \mathrm{GeV}$ for $\mathrm{e}^{-}$and 0.2 to $3 \mathrm{GeV}$ for $\mathrm{e}^{+}$were measured by the Alpha Magnetic Spectrometer (AMS) during space shuttle flight STS-91 at altitudes near $380 \mathrm{~km}$. From the origin of the leptons two distinct spectra were observed: a higher energy spectrum and a substantial second spectrum with positrons much more abundant than electrons. Tracing leptons from the second spectra shows that most of these leptons travel for an extended period of time in the geomagnetic field and that the $\mathrm{e}^{+}$and $\mathrm{e}^{-}$originate from two complementary geographic regions.
\end{abstract}

Submitted to Phys. Lett. B 


\section{Introduction}

The current understanding of the high energy lepton $\left(\mathrm{e}^{ \pm}\right)$spectra in cosmic rays is that they are dominated by an electron component. High energy electrons are believed to originate from primary acceleration sites, specifically from supernova explosions. High energy electron-positron pairs are thought to be produced from the collisions of cosmic ray hadrons and gamma rays with interstellar gas. Taken together, the expected positron to electron ratio in cosmic rays arriving at Earth is roughly $10 \%$ and it decreases with energy. This picture is based on the experimental data collected over 35 years $[1,2]$ by balloon experiments as well as phenomenological model descriptions developed over the same period [3]. These experiments were performed at altitudes of $30-40 \mathrm{~km}$. Balloon experiments have made important contributions to the understanding of primary cosmic ray spectra and the behavior of atmospheric secondary particles in the upper layer of the atmosphere.

A few pioneering satellite experiments [4] have reported data on low energy electrons and positrons trapped in the geomagnetic field. The satellite based detectors used so far, i.e. before this experiment, have not been sensitive enough to systematically study the electron and positron spectra over a broad energy range and their dependence on position and angle.

The electron spectrum observed near Earth shows a low energy drop off due to the geomagnetic cutoff. Previous measurements above the cutoff indicate that the spectrum falls off according to a power law.

The Alpha Magnetic Spectrometer (AMS) [5] is a high energy physics experiment scheduled for installation on the International Space Station. In preparation for this mission, AMS flew a precursor mission on board the space shuttle Discovery during flight STS-91 in June 1998. In this report we use the data collected to study the spectra of electrons and positrons in cosmic rays over the respective kinetic energy ranges of 0.2 to $40 \mathrm{GeV}$ and 0.2 to $3 \mathrm{GeV}$, the latter range being limited by the proton background.

The large acceptance of AMS and high statistics $\left(\sim 10^{5}\right)$ enable us to study the variation of the spectra with position and angle both above and below the geomagnetic cutoff. The accurate momentum resolution, precise trajectory reconstruction and good particle identification of AMS allow an investigation into the origin of particles below cutoff by tracking them in the geomagnetic field.

\section{The AMS detector}

The major elements of AMS as flown on STS-91 were a permanent magnet, a tracker, time of flight hodoscopes, a Cerenkov counter and anti-coincidence counters [6,7]. The permanent magnet had the shape of a cylindrical shell with inner diameter $1.1 \mathrm{~m}$, length $0.8 \mathrm{~m}$. It provided a central dipole field of 0.14 Tesla across the magnet bore and an analyzing power, $B L^{2}$, of $0.14 \mathrm{Tm}^{2}$ parallel to the magnet, or $\mathrm{z}-$, axis. The six layers of double sided silicon tracker were arrayed transverse to the magnet axis. The outer layers were just outside the magnet bore. The tracker measured the trajectory of relativistic singly charged particles with an accuracy of 20 microns in the bending coordinate and 33 microns in the non-bending coordinate, as well as providing multiple measurements of the energy loss. The time of flight system had two planes at each end of the magnet, covering the outer tracker layers. Together the four planes measured singly charged particle transit times with an accuracy of 120 psec and also yielded multiple energy loss measurements. Two layers of Aerogel threshold Cerenkov counter with an index of refraction $n=1.035$ were used to make independent velocity measurements allowing the discrimination of lower energy hadrons from electrons and positrons. A layer of anti-coincidence scintillation counters lined the inner surface of the magnet. Low energy particles were absorbed by thin carbon fiber shields. In flight the AMS positive z-axis pointed out of the shuttle payload bay. 
For this study the acceptance was restricted to events with an incident angle within $25^{\circ}$ of the positive $\mathrm{z}$-axis of AMS and data from four periods are included. In the first period the $\mathrm{z}$-axis was pointing within $1^{\circ}$ of the zenith. Events from this period are referred to as "downward" going. In the second period the $\mathrm{z}$-axis pointing was within $1^{\circ}$ of the nadir. Data from this period are referred to as "upward" going. In the third and fourth periods the AMS z-axis was pointing within $20^{\circ}$ and $45^{\circ}$ of the zenith. The orbital inclination was $51.7^{\circ}$ and the geodetic altitude during these periods ranged from 350 to $390 \mathrm{~km}$. Data taken while passing through or near the South Atlantic Anomaly were excluded from this analysis.

The response of the detector was simulated using the AMS detector simulation program, which is based on the GEANT package [8]. The effects of energy loss, multiple scattering, interactions, decays and the measured detector efficiency and resolution were included.

After the flight the AMS detector was extensively calibrated at two accelerators: at GSI, Darmstadt, with helium and carbon beams at 600 incident angles and locations and $10^{7}$ events, and at the CERN proton-synchrotron in the energy region of 2 to $14 \mathrm{GeV}$, with 1200 incident angles and locations and $10^{8}$ events. This ensured that the performance of the detector and the analysis procedure were thoroughly understood.

\section{Analysis}

Event reconstruction, analysis and spectrum unfolding are detailed in [9]. Electron candidates were specifically selected by requiring the measured particle charge to be -1 and the particle velocity to be compatible with the speed of light. Backgrounds arose from protons with wrongly measured momentum and secondary pions produced in the detector materials. The two most important cuts used to remove these backgrounds were on the $\chi^{2}$ value obtained in fitting the particle trajectory, which removed tracks with large single or multiple scattering, and on the number of hits near the reconstructed trajectory in both the tracker and time of flight scintillators.

After the above cuts were applied, the overall probability of a proton event to be accepted as an electron, estimated from Monte Carlo simulations and confirmed in the CERN test beam, was $O\left(10^{-4}\right)$ with an electron selection efficiency of $75 \%$. To further reduce the pion background only events whose track passed through the active Cerenkov counter area and, therefore, had an independent velocity measurement were accepted.

Positron candidates were selected by requiring the charge to be +1 and, as for electrons, the velocity be compatible with the speed of light and track quality cuts. In contrast to electrons, the main background for the positron sample came from proton events with poorly measured velocity. The rejection power against this background decreased rapidly with increasing proton momentum, therefore tighter quality cuts on the velocity measurements were applied. Above $1 \mathrm{GeV} / c$ protons were rejected by requiring two independent velocity measurements from the two separate Cerenkov counter layers to be compatible with the velocity of a positron. Lower energy protons were rejected by requiring the energy loss measurements in four layers of time of flight counters and six double layers of silicon tracker to be compatible with a positron. These cuts yielded an additional background rejection factor of 5 at the expense of lower positron selection efficiency. Table 1 summarizes the estimated efficiencies.

A convolution of the background rejection function with the measured proton spectra provided an energy dependent background estimation. Fig. 1 shows the measured electron and positron spectra together with the estimated background for the geomagnetic polar regions, where the background conditions were most severe. 


\begin{tabular}{|l|c|}
\hline Cut & Efficiency (\%) \\
\hline \hline Tracking Quality Cuts & $75 \pm 3$ \\
\hline Common $^{ \pm}$Velocity Cuts & $52 \pm 1$ \\
\hline Additional e ${ }^{+}$Velocity Cuts & $72 \pm 1.5$ \\
\hline \hline Total electrons & $39 \pm 1.7$ \\
\hline Total positrons & $28 \pm 1.3$ \\
\hline
\end{tabular}

Table 1: Percentage $\mathrm{e}^{ \pm}$selection efficiencies and uncertainties.

The acceptance was determined as a function of particle momentum and direction. The average acceptance was found to rise from about $0.01 \mathrm{~m}^{2} \mathrm{sr}$ at $0.15 \mathrm{GeV}$ and level off at $0.1 \mathrm{~m}^{2} \mathrm{sr}$ above $0.7 \mathrm{GeV}$ with a systematic uncertainty of $5 \%$ [9]. The incident differential spectrum was obtained from the measured spectrum by using an unfolding method based on Bayes' theorem [10] with resolution functions obtained from the simulation. These functions were confirmed at several energy points with calibration measurements in the CERN proton beams.

\section{Results and interpretation}

Fig. 2 presents the downward lepton spectra integrated over incident angles within $25^{\circ}$ of the AMS z-axis, which was within $1^{\circ}$ of the zenith. In Fig. 3 these spectra are compared with the spectra measured with upward going leptons. The measurements have been binned according to the absolute value of the corrected geomagnetic latitude [11], $\Theta_{\mathrm{M}}$ (radians), at which they were detected. The effect of the geomagnetic cutoff and the decrease in this cutoff with increasing $\Theta_{M}$ is particularly visible in the downward electron spectra. The spectra above and below cutoff differ. To understand this difference the trajectory of electrons and positrons were traced [12] back from their measured incident angle, location and momentum, through the geomagnetic field [13]. This was continued until the trajectory was traced to outside the Earth's magnetosphere or until it crossed the top of the atmosphere at an altitude of $40 \mathrm{~km}$. In a refinement from [9], the spectra from particles which were traced to originate far away from Earth are classified as "primary" and those from particles which originate in the atmosphere as "second" spectra. In practice particles below the cutoff are from the second spectra, however this classification provides a cleaner separation in the transition region.

\section{Properties of the primary lepton spectra}

Fig. 4a shows the primary lepton spectra. The spectra are in reasonable agreement with previous measurements [2]. Fig. $4 \mathrm{~b}$ shows the the energy dependence of the positron fraction, which exhibits the predominance of electrons over positrons in primary cosmic rays.

\section{Properties of the second lepton spectra}

As shown in Figs. 2 and 3, substantial second lepton spectra are observed for downward and upward going leptons at all geomagnetic latitudes below the geomagnetic cutoff. These spectra have the following properties:

(i) The second lepton spectra of Fig. 2 exhibit similar qualitative behavior to the proton spectra [9]. 
(ii) At polar latitudes the downward second spectrum of electrons is gradually obscured by the primary spectrum, whereas the second spectrum of upward going electrons is clearly observed (see Figs. 2 and 3).

(iii) For both electrons and positrons the upward and downward fluxes are nearly identical (see Fig. 3).

(iv) As seen from Fig. 5 the lepton fluxes reach a maximum at the geomagnetic equator. With increasing latitude the positron flux drops off faster than the electron flux.

In addition to the backward tracing mentioned above the leptons were also traced forward until their trajectory would have either escaped or crossed the top of the atmosphere, the location of which was taken as the particle sink. The results show that all second spectrum particles eventually re-enter the atmosphere. Defining the flight time as the sum of forward and backward tracing times, that is the interval between origin and sink, Fig. 6 shows the distribution of flight time versus energy for electrons and positrons. Both $\mathrm{e}^{+}$and $\mathrm{e}^{-}$exhibit two distinct types of trajectories:

- The horizontal bands with flight times $<0.2 \mathrm{sec}$, defined as "short-lived".

- The diagonal bands with flight times $\geq 0.2$ sec defined as "long-lived".

For $\Theta_{M}<0.3$, most $\left(75 \%\right.$ of $\mathrm{e}^{+}, 65 \%$ of $\left.\mathrm{e}^{-}\right)$leptons are long-lived.

\section{Distinct properties of the second spectra for short-lived leptons}

The trajectory tracing shows that leptons travel in cycles across the equator where the trajectories reach maximal altitude and they are reflected at the lowest points at the mid and polar latitudes. For short-lived leptons:

- From Fig. 6 one sees that the flight time is independent of lepton energy.

- The point of origin shows no longitude dependence. They do not originate from near to the geomagnetic equator, $\Theta_{M}<0.4$ (see Fig. 7a,b).

- The particle flux is independent of the shuttle attitude and is approximately isotropic (see Fig. 7c,d,e).

\section{Distinct properties of the second spectra for long-lived leptons}

- As shown in Fig. 8 long-lived $\mathrm{e}^{-}$and $\mathrm{e}^{+}$originate from well defined, complementary geographic regions. Tracing also shows that the regions of origin for positrons coincide with regions of sink for electrons and vice versa.

- Fig. 9 shows the strongly peaked distributions of the point of origin of the long-lived leptons in geomagnetic coordinates. Within the regions indicated the distributions are strongly peaked and the two diagonal bands $(A, B)$ seen in Fig. 6 for the long-lived leptons correspond to the two regions of origin $(A, B)$ marked in Figs. 8 and 9.

- The long-lived leptons are reflected across the equator hundreds of times. The number of cycles they can make before being absorbed in the atmosphere decreases with their energy. 
- As shown in Fig. 8c,d,e, the long-lived lepton flux reaches a maximum in the equatorial region where they are produced and absorbed.

- At zenith shuttle orientation, $99 \%$ of the long-lived leptons are actually detected at $\Theta_{M}<0.4$, indicating a strongly anisotropic angular distribution.

We note that the behaviour of protons and positrons is very similar (see [9]).

\section{Lepton charge ratio}

An interesting feature of the observed second lepton spectra is the predominance of positrons over electrons. In table 2 the $\mathrm{e}^{+} / \mathrm{e}^{-}$ratios grouped according to magnetic latitude region and shuttle attitude $\left(0^{\circ}, 20^{\circ}, 45^{\circ}, 180^{\circ}\right)$ are given separately for long-lived and short-lived leptons. As seen from table 2

\begin{tabular}{|c|c|c|c|c|c|}
\hline $\mathrm{e}^{+} / \mathrm{e}^{-}$ & \multicolumn{5}{|c|}{ Long-lived (flight time $\geq 0.2$ seconds) } \\
\hline Attitude & $0.0<\Theta_{\mathrm{M}}<0.2$ & $0.2<\Theta_{\mathrm{M}}<0.4$ & $0.4<\Theta_{M}<0.6$ & $0.6<\Theta_{M}<0.8$ & $0.8<\Theta_{\mathrm{M}}<1.0$ \\
\hline $0^{\circ}$ & $4.27 \pm 0.17$ & $3.26 \pm 0.37$ & $1.65 \pm 1.24$ & & \\
\hline $20^{\circ}$ & $4.15 \pm 0.39$ & $2.75 \pm 0.45$ & $2.92 \pm 1.00$ & $1.05 \pm 0.69$ & $1.46 \pm 0.42$ \\
\hline $45^{\circ}$ & $4.36 \pm 0.40$ & $3.41 \pm 0.30$ & $3.81 \pm 0.33$ & $2.27 \pm 0.18$ & $1.28 \pm 0.16$ \\
\hline $180^{\circ}$ & $4.27 \pm 0.25$ & $4.25 \pm 0.65$ & & & \\
\hline 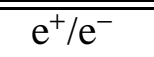 & \multicolumn{5}{|c|}{ Short-lived (flight time $<0.2$ seconds) } \\
\hline Attitude & $0.0<\Theta_{M}<0.2$ & $0.2<\Theta_{M}<0.4$ & $0.4<\Theta_{M}<0.6$ & $0.6<\Theta_{M}<0.8$ & $0.8<\Theta_{M}<1.0$ \\
\hline $0^{\circ}$ & $3.08 \pm 0.35$ & $2.43 \pm 0.19$ & $1.35 \pm 0.11$ & $1.10 \pm 0.11$ & $0.83 \pm 0.10$ \\
\hline $20^{\circ}$ & $2.83 \pm 0.67$ & $2.23 \pm 0.37$ & $1.95 \pm 0.28$ & $1.48 \pm 0.22$ & $0.94 \pm 0.18$ \\
\hline $45^{\circ}$ & $3.22 \pm 0.44$ & $2.18 \pm 0.32$ & $2.01 \pm 0.32$ & $1.08 \pm 0.12$ & $0.93 \pm 0.19$ \\
\hline $180^{\circ}$ & $4.84 \pm 0.81$ & $2.79 \pm 0.28$ & $1.45 \pm 0.18$ & $1.17 \pm 0.21$ & $0.68 \pm 0.27$ \\
\hline
\end{tabular}

Table 2: Lepton charge ratio versus magnetic latitude for the shuttle attitudes $0^{\circ}, 20^{\circ}, 45^{\circ}$ and $180^{\circ}$ for long-lived and short-lived particles.

the ratios:

- Depend at most weakly on the shuttle orientation.

- The ratios for short- and long-lived leptons behave differently. For short-lived leptons the $\mathrm{e}^{+} / \mathrm{e}^{-}$ ratio is maximal at the magnetic equator where it reaches a value of $\sim 3$ whereas for long-lived leptons the ratio is higher, $\gtrsim 4$ at the magnetic equator, and less dependent on latitude.

- The energy dependence of the $\mathrm{e}^{+} / \mathrm{e}^{-}$ratio for $0^{\circ}$ attitude and $\Theta_{\mathrm{M}}<0.3$ is shown in Fig. 10. As seen, short-lived and long-lived leptons behave differently. For short-lived leptons the ratio does not depend on the particle energy in the range 0.2 to $3 \mathrm{GeV}$ but for long-lived leptons the ratio does depend on the lepton energy, reaching a maximum value of $\sim 5$.

The combined (short- and long-lived, all attitudes) dependence on $\Theta_{M}$ of the ratio for all second spectra particles is shown in Fig. 11. 


\section{Acknowledgements}

The support of INFN, Italy, ETH-Zürich, the University of Geneva, the Chinese Academy of Sciences, Academia Sinica and National Central University, Taiwan, the RWTH-Aachen, Germany, the University of Turku, the University of Technology of Helsinki, Finland, the U.S. DOE and M.I.T., CIEMAT, Spain, LIP, Portugal and IN2P3, France, is gratefully acknowledged.

The success of the first AMS mission is due to many individuals and organizations outside of

the collaboration. The support of NASA was vital in the inception, development and operation of the experiment. Support from the Max-Plank Institute for Extraterrestrial Physics, from the space agencies of Germany (DLR), Italy (ASI), France (CNES) and China and from CSIST, Taiwan also played important parts in the success of AMS.

\section{References}

[1] R.R. Daniel and S.A. Stephens, Phys. Rev. Lett. 15 (1965) 769; C.J. Bland et al., Phys. Rev. Lett. 17 (1966) 813; S.D. Verma, J. Geophys. Res. 72 (1967) 915; C.J. Bland et al., Nouvo Cim. LV B (1968) 451; B. Agrinier et al., Nouvo Cim.Lett. 1 (1969) 54; J.L. Fanselow et al., ApJ 158 (1969) 771; J. Daugherty et al., ApJ 198 (1975) 493; A. Buffington et al., ApJ 199 (1975) 669; R. Hartman and C. Pellerin, ApJ 204 (1976) 927; K.K. Tang, ApJ 278 (1984) 881; R.L. Golden et al., ApJ 287 (1985) 662; D. Muller and K. Tang, ApJ 312 (1987) 183; G. Barbiellini et al., Astronomy and Astrophysics 309 (1996) L15; R.L. Golden et al., ApJ 457 (1996) L103; S.W. Barwick et al., ApJ 482 (1997) L191; S.W. Barwick et al., J. Geophys. Res. 103 (1998) 4817; S. Torii et al., Proc. 26th ICRC 3 (1999) 53; M. Boezio et al., Proc. 26th ICRC 3 (1999) 57; S. Coutu et al., Astropart.Phys. 11 (1999) 429.

[2] R.L. Golden et al., ApJ 436 (1994) 769; S.W. Barwick et al., ApJ 498 (1998) 779; M.A. DuVernois et al., Proc. 26th ICRC 3 (1999) 49.

[3] O.A. Bogdanova et al., 15 ICRC Plovdiv 3 (1977) 176; M. Giler et al., J.Phys.A:Math.Gen. 10 (1977) 843; R.J. Protheroe, ApJ 254 (1982) 391; W.R. Webber, 20 ICRC 2 (1987) 80; I.V. Moskalenko and A.W. Strong, ApJ 493 (1998) 694.

[4] J.B. Cladis et al., J. Geophys. Res. 66 (1961) 2297; L.V. Kurnosova et al., 15 ICRC Plovdiv 4 (1977) 185; R.N. Basilova et al., 16 ICRC Kyoto 3 (1979) 150; N.L. Grigorov et al., Dokl.Akad.Nauk SSSR 282 (1985) 81; Yu.E. Efimov et al., Chechoslovak Journ. of Phys. 35 (1985) 1371; S.A. Voronov et al., Izv.Vysshikh Uchebn.Zavedenii,Fizika 9 (1986) 1924; S.A. Voronov et al., Geomagnetism and Aeronomy 27 (1987) 424; A.F. Iydin et al., Geomagnetism and Aeronomy 28 (1988) 103; S.V. Koldashov et al., 24 ICRC Roma 4 (1995) 993; A.M. Galper et al., 25 ICRC Durban 4 (1997) 333.

[5] S. Ahlen et al., Nucl. Inst. Meth. A350 (1994) 351.

[6] G.M. Viertel and M. Capell, Nucl. Inst. Meth. A419 (1998) 295-299.

[7] AMS Collaboration, J. Alcaraz et al., Phys. Lett. B461 (1999) 387-396.

[8] R. Brun et al., GEANT 3, CERN DD/EE/84-1 (Revised, 1987); P.A. Aamio et al., FLUKA Users Guide, CERN TIS-RP-190 (1990).

[9] AMS Collaboration, J. Alcaraz et al., Phys. Lett. B472 (2000) 215-226. 
[10] A. Kondor, Nucl. Inst. Meth. 216 (1983) 177; G. D’Agostini, Nucl. Inst. Meth. A362 (1995) 487.

[11] A. Brekke, Physics of the Upper Polar Atmosphere, pp. 127-145, (Wiley, 1997).

[12] Y.L. Chuang et al., Chinese Journal of Physics, in preparation (2000); N. Zographos, ETHZ-IPP 99-04 (1999).

[13] N.A. Tsyganenko and A.V. Usmanov, Planet. Space Sci. 30 (1982) 985-998; N.A. Tsyganenko et al., Software for Computations of Geomagnetic Field and Related Coordinate Systems, Soviet Geophysical Committee, Special Report (1987); N.A. Tsyganenko, Planet. Space Sci. 35 (1987) 1347-1358; N.A. Tsyganenko, Planet. Space Sci. 37 (1989) 5-20; N.A. Tsyganenko, J. Geophys. Res. 100 (1995) 5599-5612; N.A. Tsyganenko and D.P. Stern, J. Geophys. Res. 101 (1996) 27187-27198; R.L. Langel, Chairman, IAGA Div. V working group 8, J. Geomag. Geoelectr. 47 (1995) 1251-1261; G. Gustafsson, N.E. Papitashvili and V.O. Papitashvili, J. Atmos. Terr. Phys. 54 (1992) 1609-1631.

\section{The AMS Collaboration}

J.Alcaraz ${ }^{y}$ B.Alpat, ${ }^{a c}$ G.Ambrosi ${ }^{r}$ H.Anderhub, ${ }^{a g}$ L.Ao, ${ }^{g}$ A.Arefiev ${ }^{a b}$ P.Azzarello, ${ }^{r}$ E.Babucci, ${ }^{a c}$ L.Baldini ${ }^{j, l}$ M.Basile, D.Barancourt, ${ }^{s}$ F.Barao, ${ }^{w, v}$ G.Barbier, G.Barreira, ${ }^{w}$ R.Battiston, ${ }^{a c}$

R.Becker, U.Becker, L.Bellagamba, P.Bénér, J.Berdugo, ${ }^{l}$ P.Berges, B.Bertuccia, ${ }^{l}{ }^{l}$ A.Biland, ${ }^{a g}$

S.Bizzaglia, ${ }^{a c}$ S.Blasko, ${ }^{a c}$ G.Boella ${ }^{z}$ M.Boschini, M.Bourquin ${ }^{r}$, L.Brocco ${ }^{j}$, G.Bruni ${ }^{j}$, M.Buenerd${ }^{s}$ J.D.Burger, W.J.Burger, ${ }^{a c}$ X.D.Cai, C.Camps, P.Cannarsa, ${ }^{a g}$ M.Capell, D.Casadei, J.Casaus, G.Castellini, C.Cecchi, ${ }^{a c}$ Y.H.Chang, ${ }^{m}$ H.F.Chen ${ }^{t}$ H.S.Chen, Z.G.Chen ${ }^{g}$ N.A.Chernoplekov, ${ }^{a a}$ T.H.Chiueh ${ }^{m}{ }^{\text {Y.L.Chuang, }}{ }^{a d}{ }^{\text {F.Cindolo }}{ }^{j}$, V.Commichau, ${ }^{b}$ A.Contin ${ }^{j}$, P.Crespo, ${ }^{w}$ M.Cristinziani ${ }^{r}$


J.Engelberg, ${ }^{u}$ F.J.Eppling, T.Eronen, ${ }^{a f}$ G.Esposito, ${ }^{a c}$ P.Extermann, ${ }^{r}$ J.Favier, E.Fiandrini, ${ }^{a c}$ P.H.Fisher, G.Fluegge ${ }^{b}$, N.Fouque, Yu.Galaktionov, ${ }^{a b, l}$ M.Gervasi, P.Giusti, D.Grandi, O.Grimm, ${ }^{j}{ }^{j}$ W.Q.Gu, ${ }^{h}$ K.Hangarter, A.Hasan, ${ }^{a g}$ V.Hermel, H.Hofer, ${ }^{a g}$ M.A.Huang, ${ }^{a d}$ W.Hungerford, ${ }^{a g}$ M.Ionica, ${ }^{a c, 1}$ R.Ionica, ${ }^{a c, 1}$ M.Jongmanns, ${ }^{a g}$ K.Karlamaa, ${ }^{u}$ W.Karpinski, G.Kenney, ${ }^{a g}$ J.Kenny, ${ }^{a c}$ W.Kim, ${ }^{a e}$ A.Klimentov, ${ }^{l, a b}$ R.Kossakowski, V.Koutsenko, ${ }^{l, a b}$ M.Kraeber, ${ }^{a g}$ G.Laborie, T.Laitinen, ${ }^{a f}$ G.Lamanna, G.Laurenti, A.Lebedev, ${ }^{l}$ S.C.Lee, ${ }^{a d}$ G.Levi, P.Levtchenko, ${ }^{a c, 2}$ C.L.Liu, ${ }^{x}$ H.T.Liu, ${ }^{i}$ I.Lopes, ${ }^{n}$ G.Lu, ${ }^{g}$ Y.S.Lu, K.Lübelsmeyer, D.Luckey, W.Lustermann, ${ }^{a}{ }^{i}$ C.Maña ${ }^{y}$ A.Margotti ${ }^{j}$, F.Mayet$^{s}$, R.R.McNeil ${ }^{e}$ B.Meillon, ${ }^{s}$ M.Menichelli, ${ }^{a c}$ A.Mihul, ${ }^{k}$ A.Mourao, ${ }^{v}$ A.Mujunen, ${ }^{u}$ F.Palmonari, A.Papia ${ }^{a c}$ I.H.Park ${ }^{a e}$ M.Pauluzzi, ${ }^{a c}$ F.Pauss, ${ }^{a g}$ E.Perrin ${ }^{r}$ A.Pesci, A.Pevsner, ${ }^{d}$ M.Pimenta, ${ }^{w, v}$ V.Plyaskin, ${ }^{a b}$ V.Pojidaev, ${ }^{a b}$ V.Postolache, ${ }^{a c, 1}$ N.Produit, P.G.Rancoita ${ }^{z}$ D.Rapin, F.Raupach, D.Ren, ${ }^{a g}$ Z.Ren, ${ }^{a}{ }^{a}$ M.Ribordy, J.P.Richeux, E.Riihonen, ${ }^{a f}$ J.Ritakari, ${ }^{u}$ U.Roeser, ${ }^{a g}$ C.Roissin, ${ }^{s}$ R.Sagdeev, ${ }^{o}$ G.Sartorelli, A.Schultz von Dratzig, ${ }^{a}$ G.Schwering, ${ }^{a}$ G.Scolieri, ${ }^{a c}$ E.S.Seo, V.Shoutko, E.Shoumilov, ${ }^{a b}$ R.Siedling, D.Son, ${ }^{a e}$ T.Song, M.Steuer, G.S.Sun, H.Suter, ${ }^{l}{ }^{l}$ X.W.Tang, Samuel C.C.Ting, ${ }^{l}$, S.M.Ting, M.Tornikoski, J.Torsti, ${ }^{a f}$ J.Trümper ${ }^{q}$ J.Ulbricht, ${ }^{a g}$ S.Urpo, I.Usoskin, E.Valtonen, ${ }^{a f}$ J.Vandenhirtz, F.Velcea, ${ }^{a c, 1}$ E.Velikhov, ${ }^{a a}$ B.Verlaat, ${ }^{a g, 3}{ }^{\prime}$ I.Vetlitsky, ${ }^{a b}$ F.Vezzu, ${ }^{s}$ J.P.Vialle, G.Viertel, ${ }^{a g}$ D.Vité, H.Von Gunten, ${ }^{a g}$ S.Waldmeier Wicki, ${ }^{a g}$ W.Wallraff, B.C.Wang, J.Z.Wang, ${ }^{g}$ Y.H.Wang, ${ }^{a d}$ K.Wiik, C.Williams, ${ }^{j}$ S.X.Wu, ${ }^{l, m}$ P.C.Xia, J.L.Yan ${ }^{g}$ L.G.Yan, ${ }^{h}$ C.G.Yang, M.Yang ${ }^{i}$, S.W.Ye, ${ }^{t, 4}{ }^{i}$ P.Yeh, ${ }^{a d}{ }^{2}$ Z.Z.Xu, H.Y.Zhang, Z.P.Zhang, D.X.Zhao, ${ }^{h}$ G.Y.Zhu, W.Z.Zhu, H.L.Zhuang, A.Zichichi, B.Zimmermann. ${ }^{i g}$

${ }^{a}$ I. Physikalisches Institut, RWTH, D-52056 Aachen, Germany ${ }^{5}$

${ }^{b}$ III. Physikalisches Institut, RWTH, D-52056 Aachen, Germany ${ }^{6}$ 
${ }^{c}$ Laboratoire d'Annecy-le-Vieux de Physique des Particules, LAPP, F-74941 Annecy-le-Vieux CEDEX, France

${ }^{e}$ Louisiana State University, Baton Rouge, LA 70803, USA

$d$ Johns Hopkins University, Baltimore, MD 21218, USA

${ }^{f}$ Center of Space Science and Application, Chinese Academy of Sciences, 100080 Beijing, China

$g$ Chinese Academy of Launching Vehicle Technology, CALT, 100076 Beijing, China

$h$ Institute of Electrical Engineering, IEE, Chinese Academy of Sciences, 100080 Beijing, China

${ }^{i}$ Institute of High Energy Physics, IHEP, Chinese Academy of Sciences, 100039 Beijing, China ${ }^{6}$

$j$ University of Bologna and INFN-Sezione di Bologna, I-40126 Bologna, Italy

${ }^{k}$ Institute of Microtechnology, Politechnica University of Bucharest and University of Bucharest, R-76900 Bucharest, Romania

$l$ Massachusetts Institute of Technology, Cambridge, MA 02139, USA

$m$ National Central University, Chung-Li, Taiwan 32054

${ }^{n}$ Laboratorio de Instrumentacao e Fisica Experimental de Particulas, LIP, P-3000 Coimbra, Portugal

$o$ University of Maryland, College Park, MD 20742, USA

$p$ INFN Sezione di Firenze, I-50125 Florence, Italy

$q$ Max-Plank Institut fur Extraterrestrische Physik, D-85740 Garching, Germany

$r$ University of Geneva, CH-1211 Geneva 4, Switzerland

$s$ Institut des Sciences Nucleaires, F-38026 Grenoble, France

${ }^{t}$ Chinese University of Science and Technology, USTC, Hefei, Anhui 230 029, China ${ }^{7}$

$u$ Helsinki University of Technology, FIN-02540 Kylmala, Finland

$v$ Instituto Superior Técnico, IST, P-1096 Lisboa, Portugal

${ }^{w}$ Laboratorio de Instrumentacao e Fisica Experimental de Particulas, LIP, P-1000 Lisboa, Portugal

${ }^{x}$ Chung-Shan Institute of Science and Technology, Lung-Tan, Tao Yuan 325, Taiwan

${ }^{y}$ Centro de Investigaciones Energéticas, Medioambientales y Tecnologícas, CIEMAT, E-28040 Madrid, Spain $^{7}$

$z$ INFN-Sezione di Milano, I-20133 Milan, Italy

aa Kurchatov Institute, Moscow, 123182 Russia

$a b$ Institute of Theoretical and Experimental Physics, ITEP, Moscow, 117259 Russia

ac INFN-Sezione di Perugia and Universitá Degli Studi di Perugia, I-06100 Perugia, Italy ${ }^{8}$

ad Academia Sinica, Taipei 11529, Taiwan

ae Kyungpook National University, 702-701 Taegu, Korea

af University of Turku, FIN-20014 Turku, Finland

ag Eidgenössische Technische Hochschule, ETH Zürich, CH-8093 Zürich, Switzerland

1 Permanent address: HEPPG, Univ. of Bucharest, Romania.

2 Permanent address: Nuclear Physics Institute, St. Petersburg, Russia.

3 Now at European Laboratory for Particle Physics, CERN, CH-1211 Geneva 23, Switzerland.

4 Now at National Institute for High Energy Physics, NIKHEF, NL-1009 DB Amsterdam, The Netherlands.

5 Supported by ETH Zürich.

6 Supported by the Deutsches Zentrum für Luft- und Raumfahrt, DLR.

7 Supported by the National Natural Science Foundation of China.

8 Also supported by the Comisión Interministerial de Ciencia y Tecnología.

9 Also supported by the Italian Space Agency. 




Figure 1: The primary $\mathrm{e}^{ \pm}$fluxes and background in the geomagnetic polar region $\left(\Theta_{M}>0.9\right)$. 


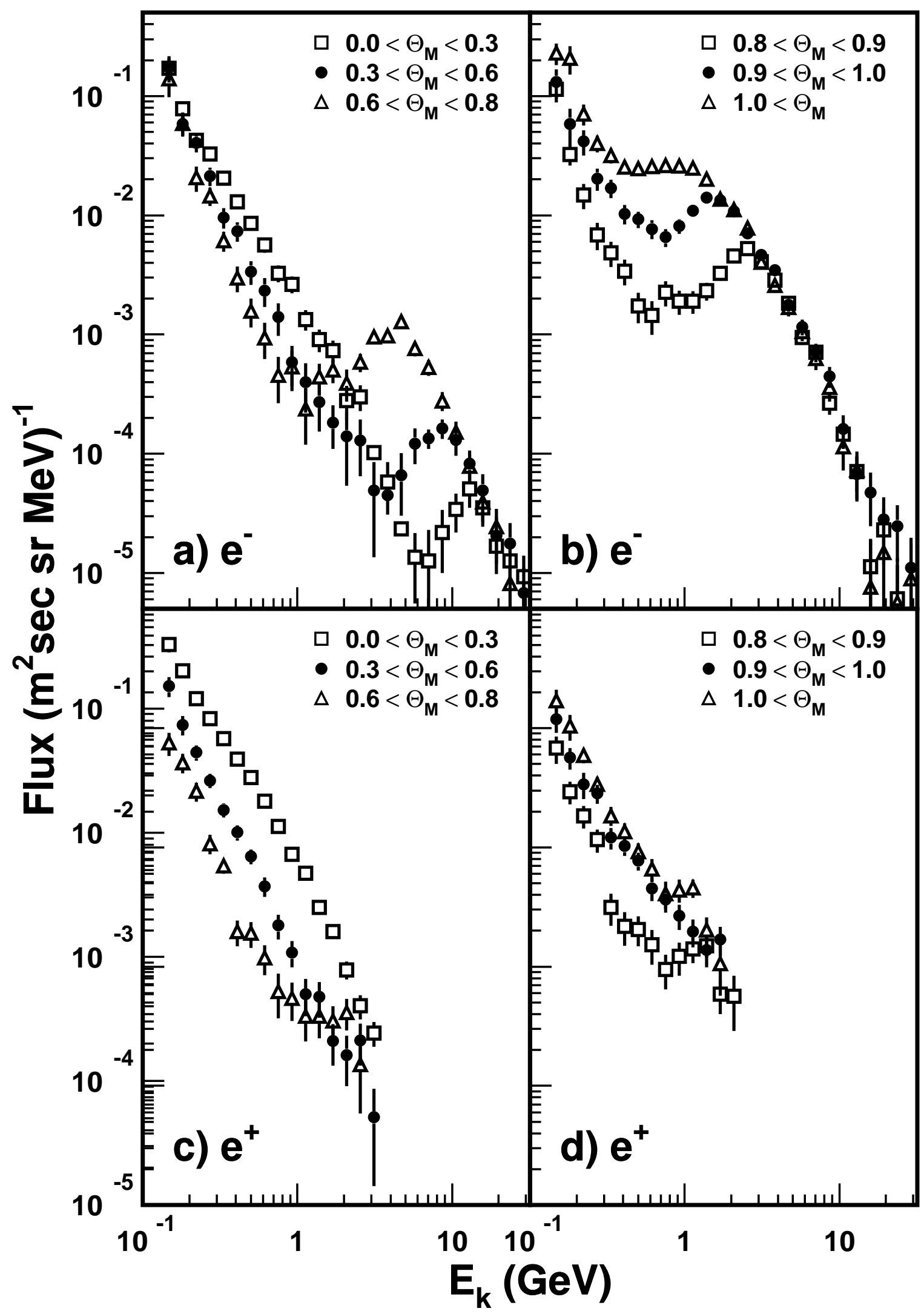

Figure 2: (a,b) Flux spectra for downward going electrons and (c,d) positrons, separated according to the geomagnetic latitude at which they were detected. 


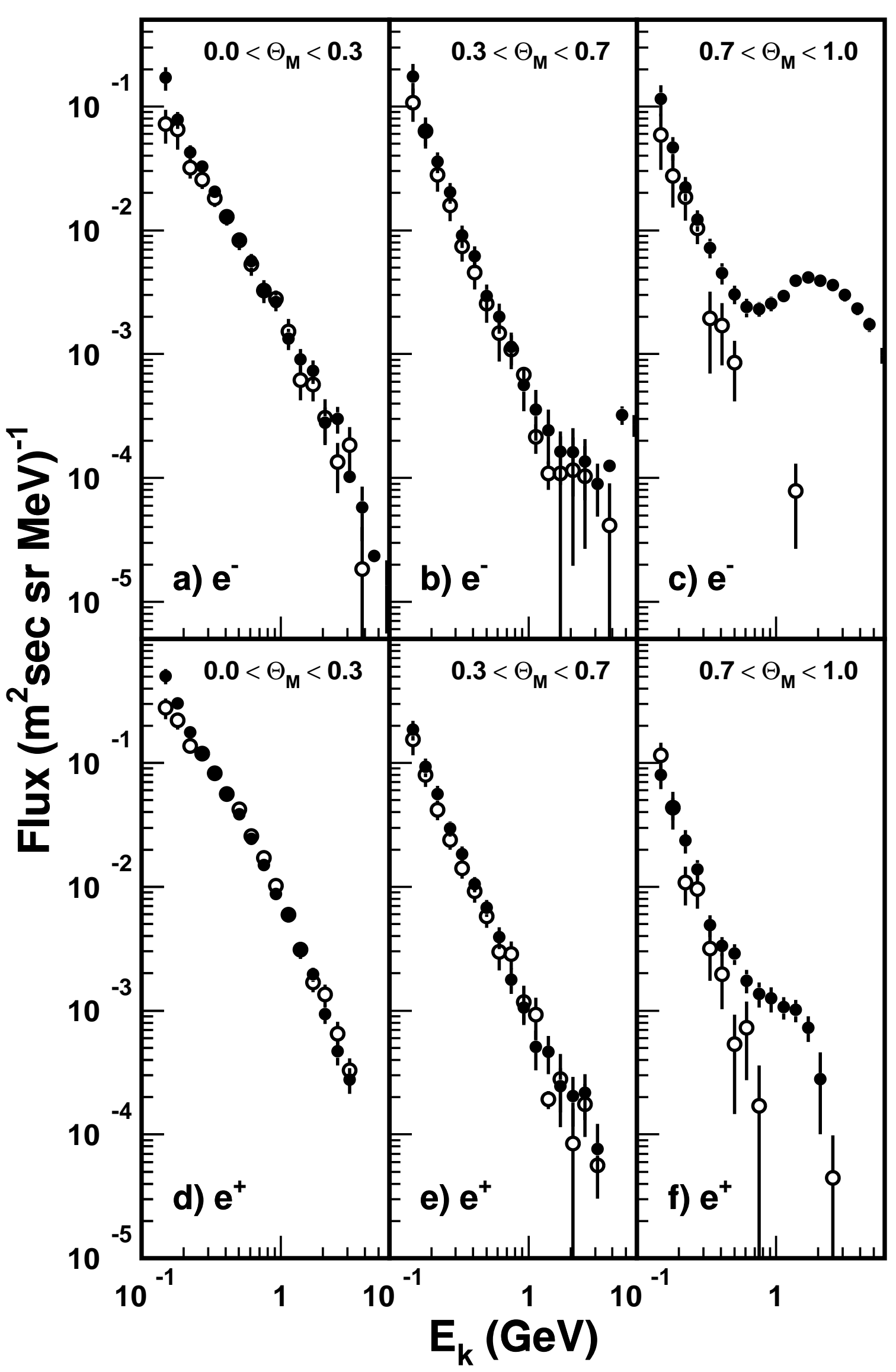

Figure 3: (a,b,c) Flux spectra for downward (full circles) and upward (open circles) going electrons and $(\mathrm{d}, \mathrm{e}, \mathrm{f})$ positrons, separated according to the geomagnetic latitude, $\Theta_{\mathrm{M}}$, at which they were detected. 

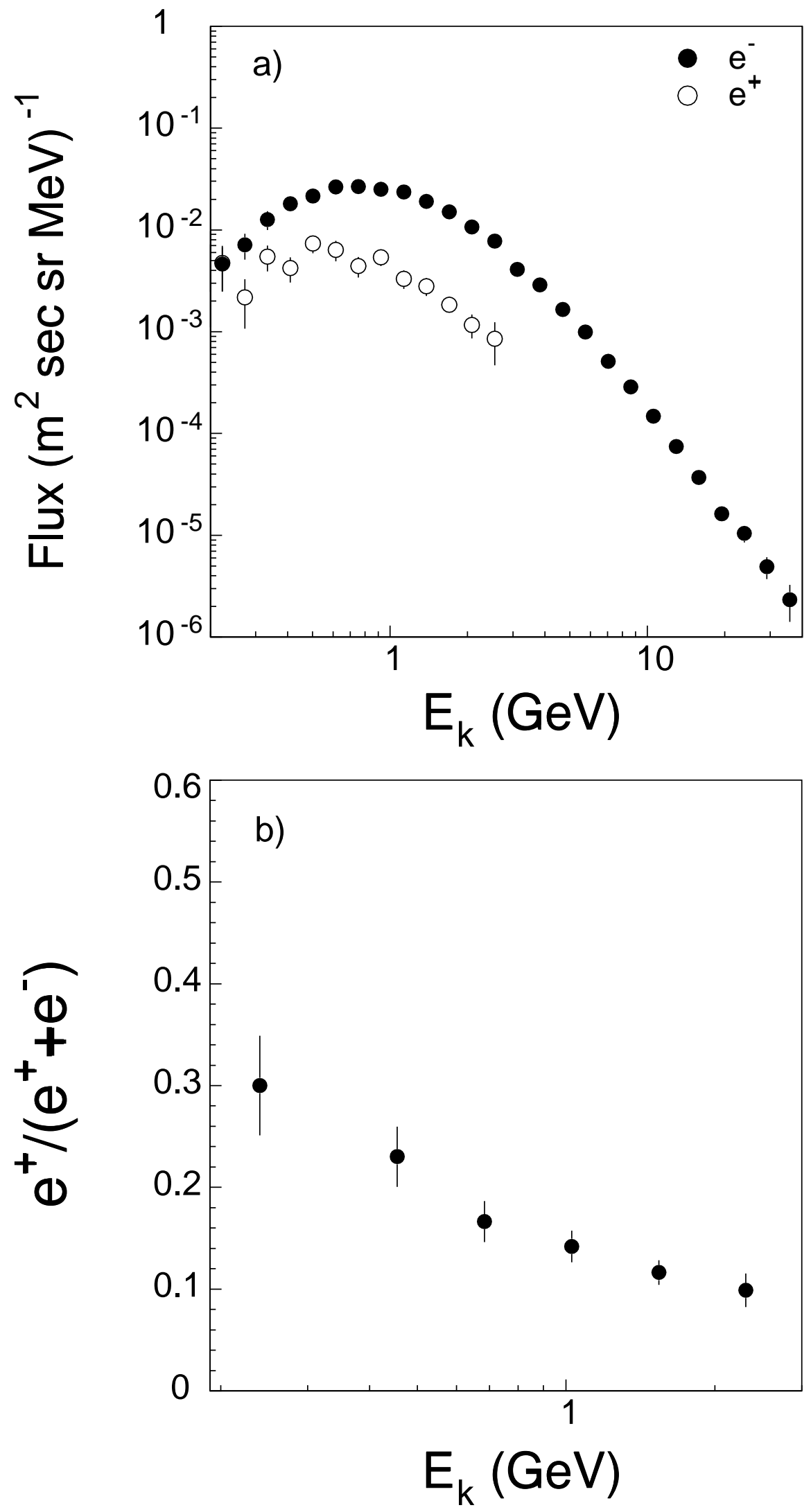

Figure 4: (a) Flux spectra for primary leptons. Particle direction within $25^{\circ}$ of zenith. (b) Positron fraction for primary leptons versus energy. 


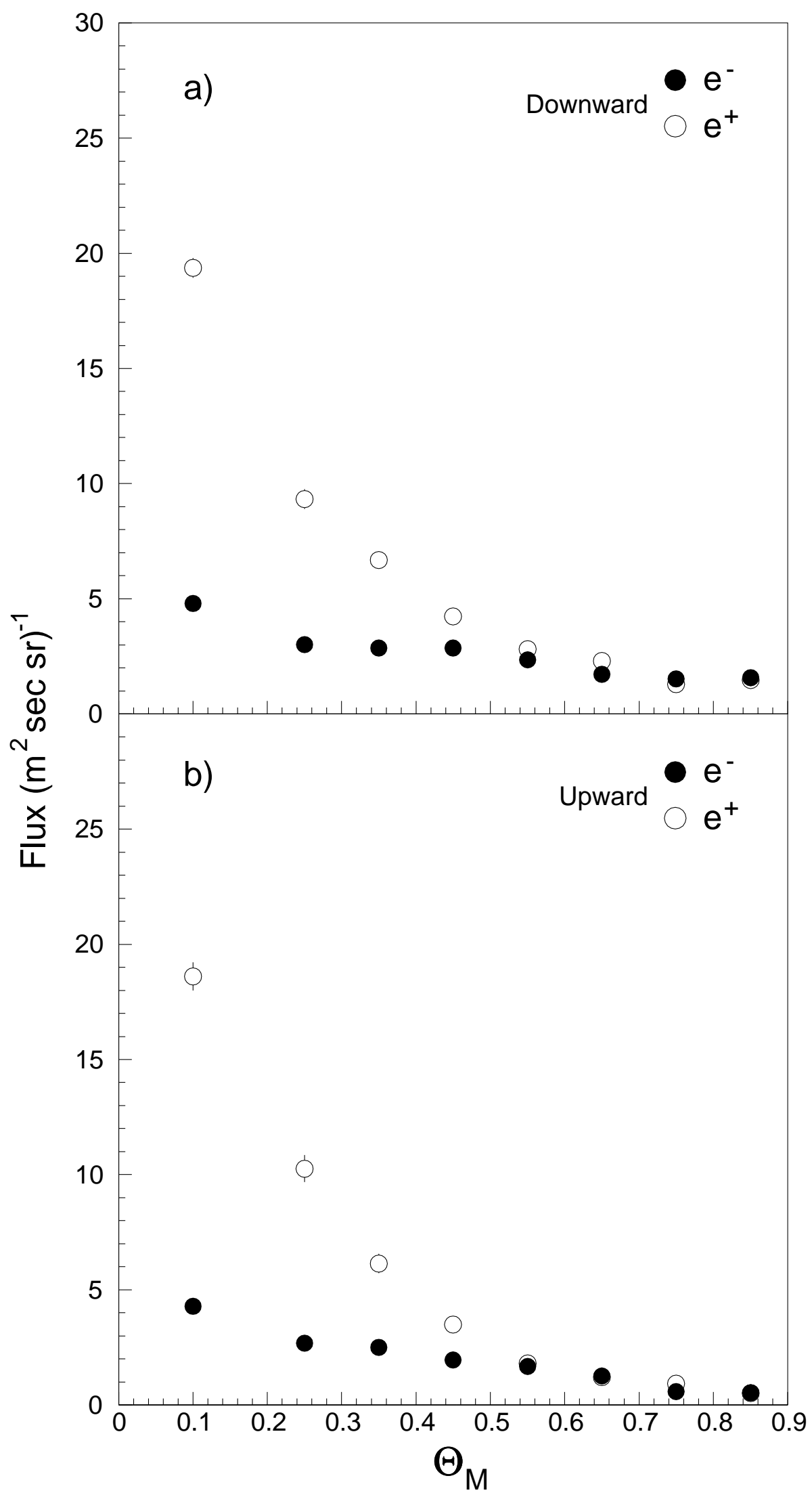

Figure 5: Properties of second lepton spectra flux: (a) downward and (b) upward going electrons and positrons as functions of the geomagnetic latitude, $\Theta_{M}$, at which they were detected integrated over the range $0.2-2.5 \mathrm{GeV}$. 


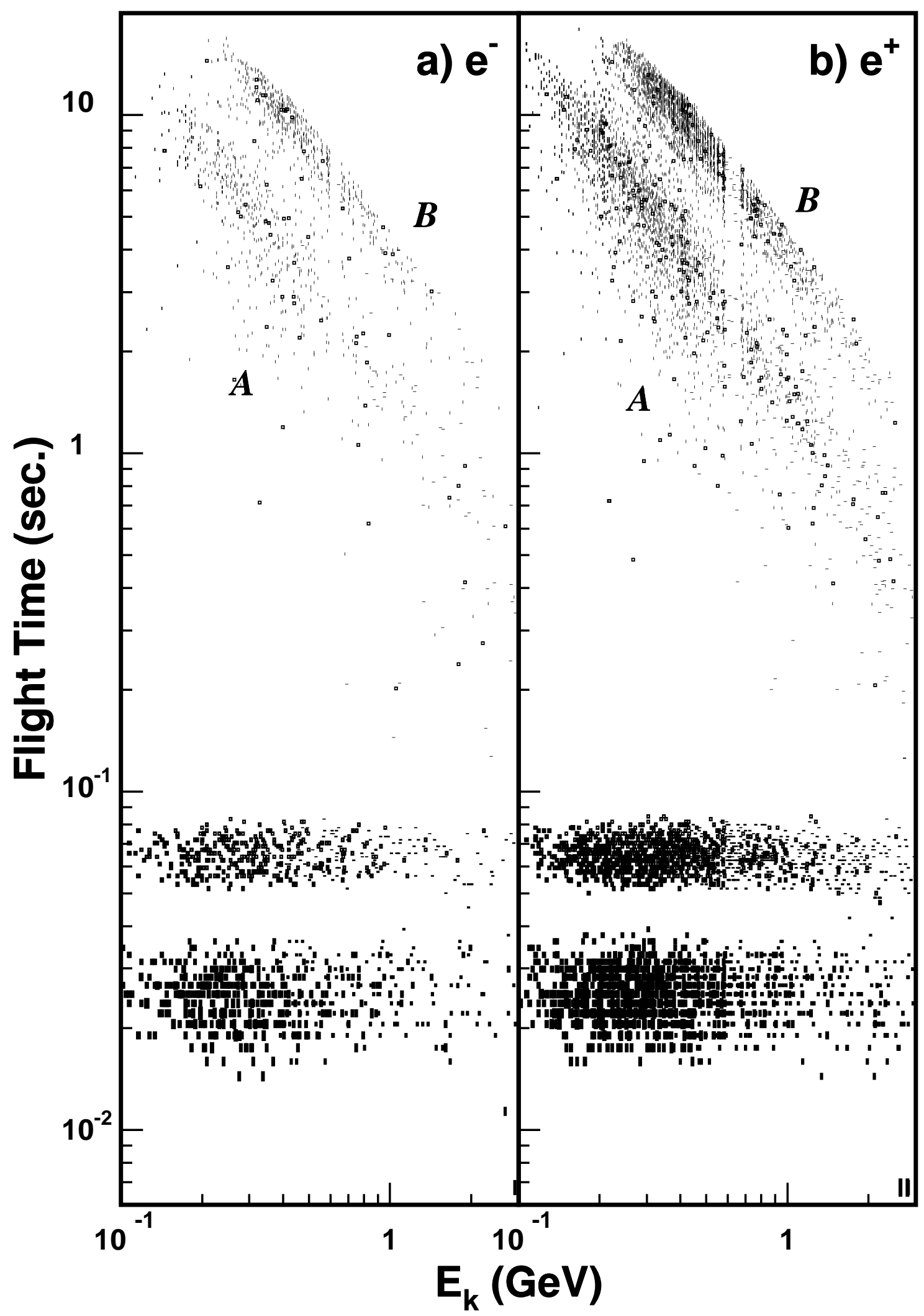

Figure 6: The flight time versus energy from the tracing of leptons detected in the region $\Theta_{M}<0.3$. From the flight time distribution there are two distinct types of trajectories: For "short-lived", flight times $<0.2 \mathrm{sec}$, the flight time is independent of lepton energy. For "long-lived", flight times $\geq$ $0.2 \mathrm{sec}$, there are two bands $A$ and $B$. In both $A$ and $B$ the flight time depends on energy: it decreases with increasing energy. 

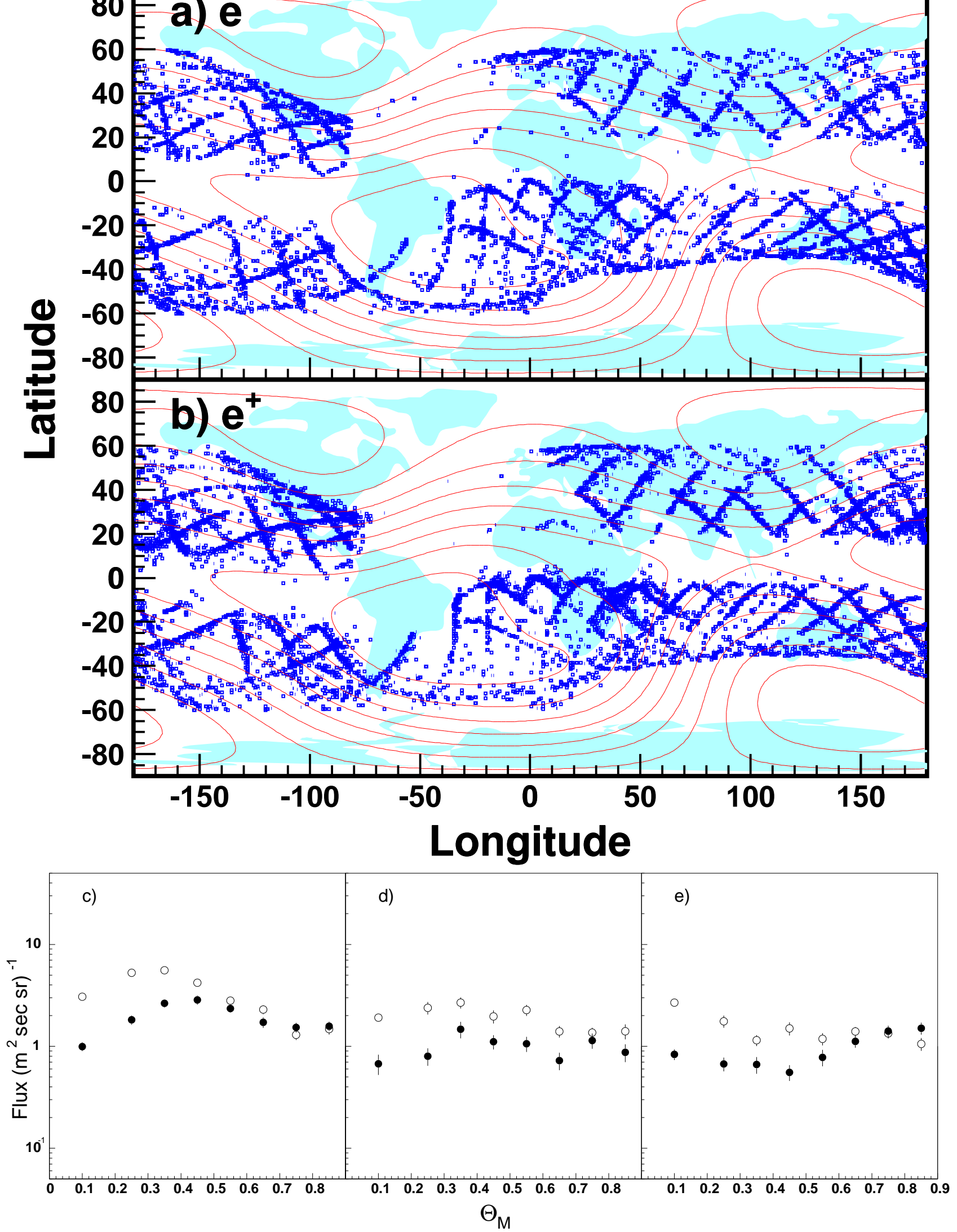

Figure 7: Properties of short-lived second spectra leptons $(<3 \mathrm{GeV})$ : (a) The geographic origin of electrons and (b) positrons. Note that the point of origin shows no longitudinal dependence and that the short-lived leptons do not originate from the region $\Theta_{M}<0.4$. The lines indicate the geomagnetic field contours at $380 \mathrm{~km}$. (c) The $\mathrm{e}^{-}$(full circles) and $\mathrm{e}^{+}$(open circles) fluxes integrated over the range 0.2-2.5 GeV as a function of magnetic latitude for zenith, (d) $20^{\circ}$ and (e) $45^{\circ}$ shuttle attitude. 

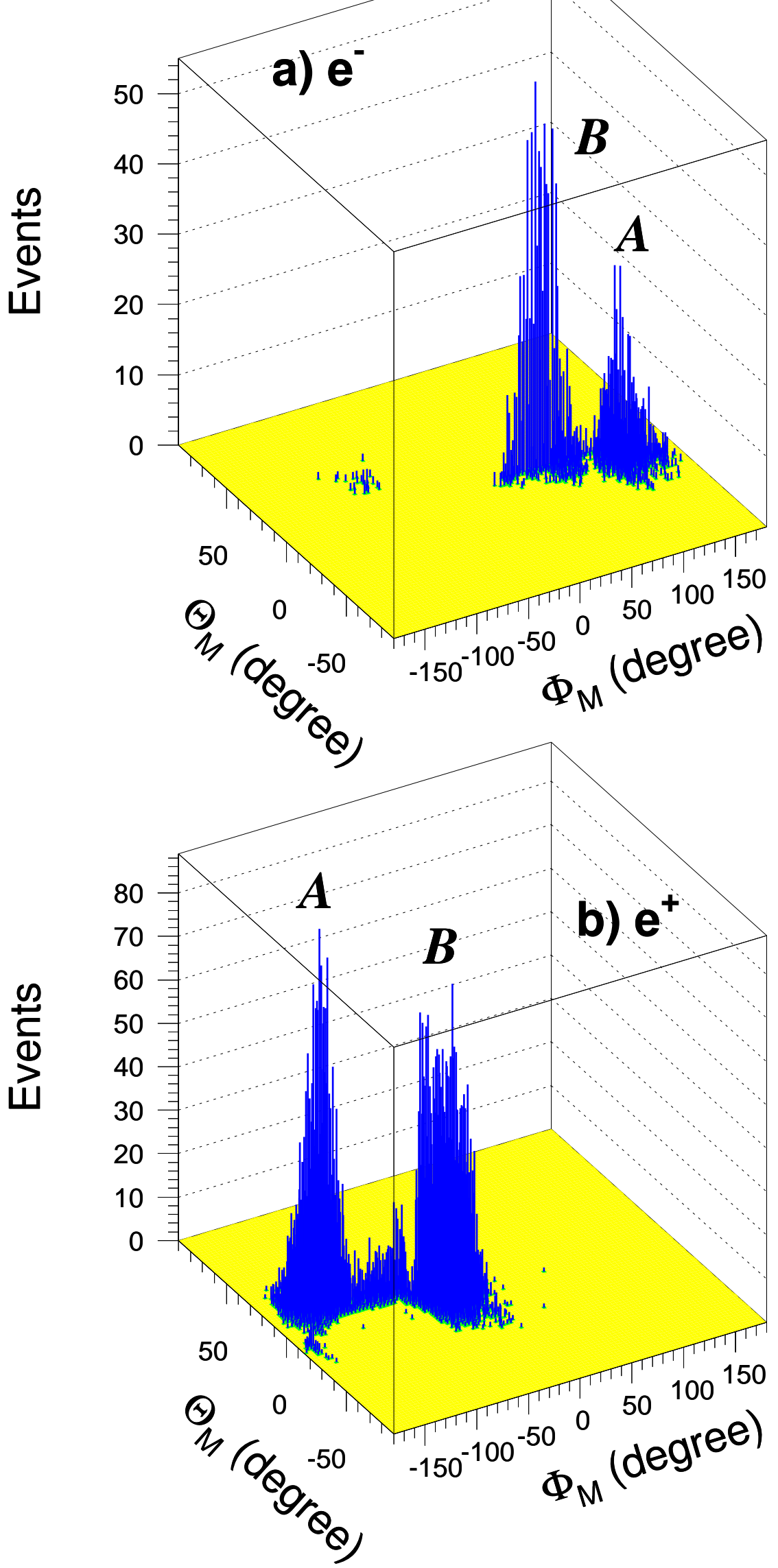

Figure 9: Property of second spectra: The point of origin of long-lived leptons with energies $<3 \mathrm{GeV}$ and $\Theta_{\mathrm{M}}<0.7$ in geomagnetic coordinates. The regions $A$ and $B$ correspond to those in Fig. 8 and the bands marked $A$ and $B$ in Fig. 6 . 


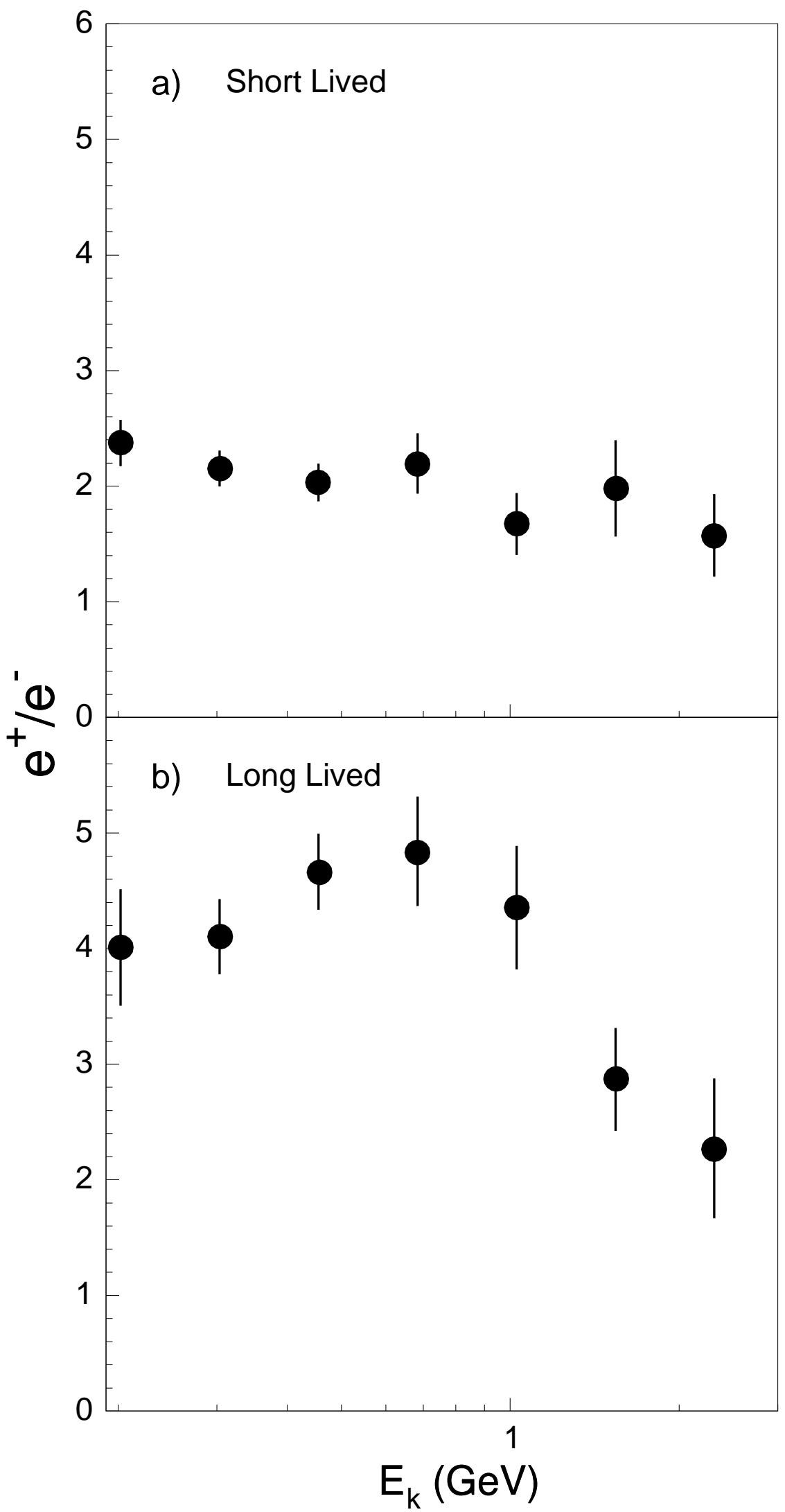

Figure 10: Property of second spectra: The $\mathrm{e}^{+} / \mathrm{e}^{-}$ratio as a function of energy for (a) short-lived and (b) long-lived particles. Shuttle attitude $0^{\circ}$ and $\Theta_{\mathrm{M}}<0.3$. 


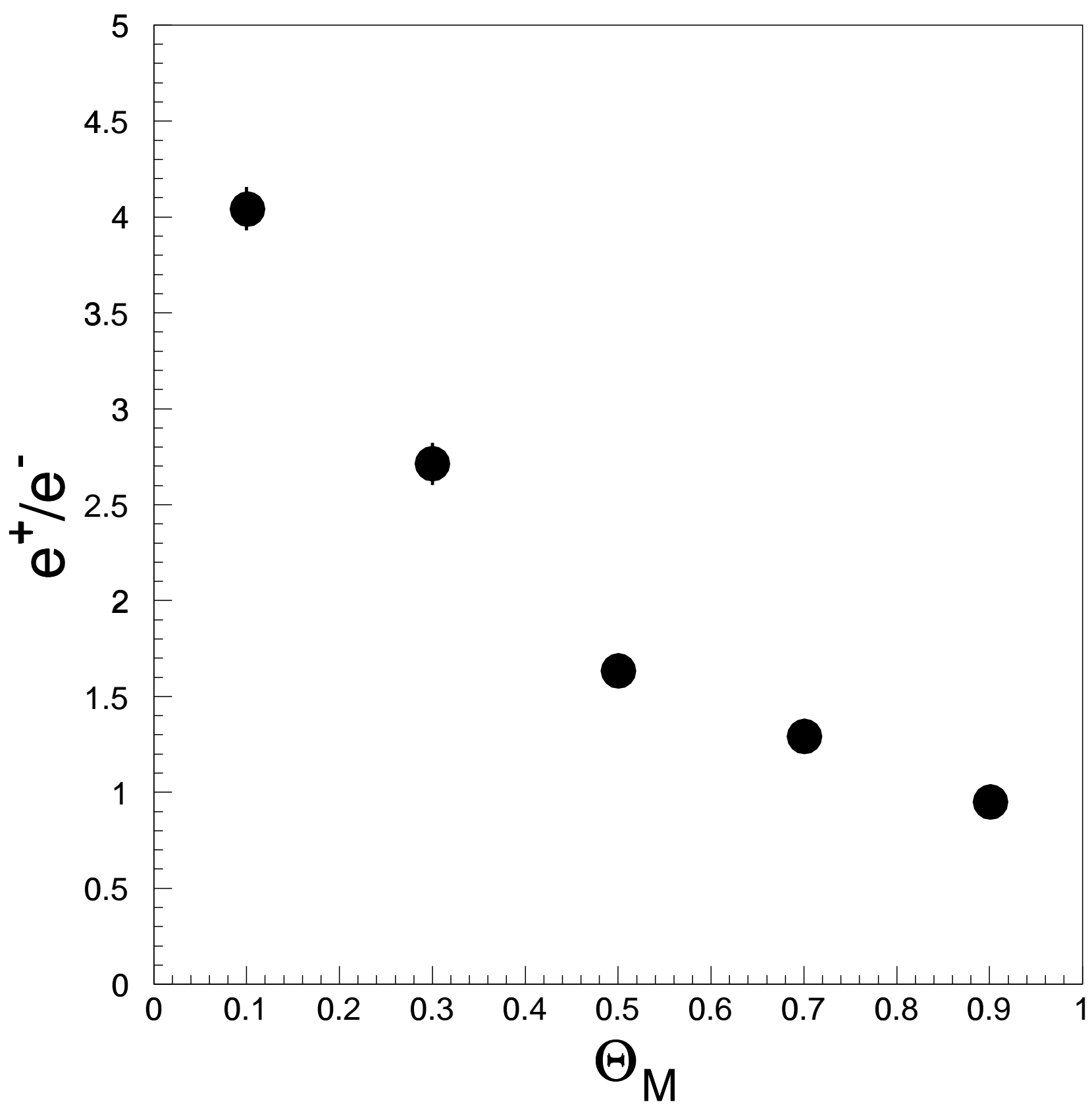

Figure 11: Property of second spectra: The $\mathrm{e}^{+} / \mathrm{e}^{-}$ratio as a function of magnetic latitude integrated over the range $0.2-2.5 \mathrm{GeV}$ and combined for short-lived and long-lived leptons independent of shuttle attitude. 